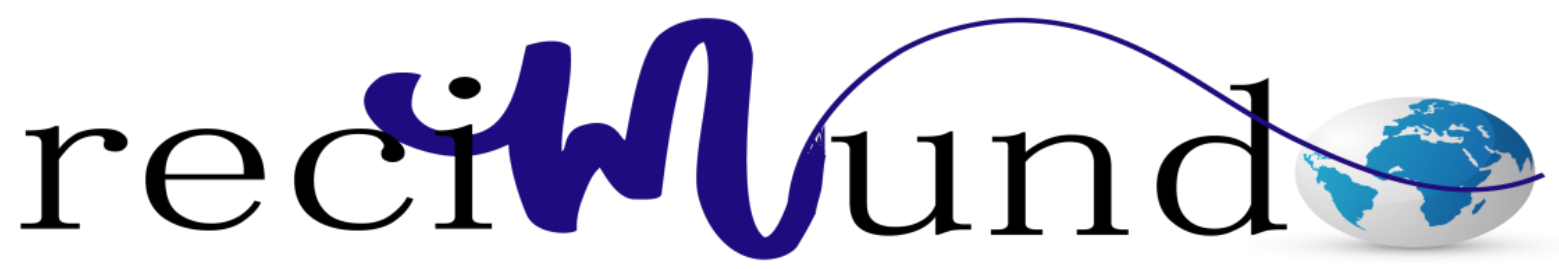

Revista Científica Mundo de la Investigación y el Conocimiento

Kerly Bachita Zambrano Macías a; María Paulina Tórres Vasconez ${ }^{\text {b; }}$ Anay Teresita Brito Martínez ${ }^{\mathrm{c}}$; Yesica Yadira Pazmiño Mera ${ }^{\mathrm{d}}$

Psicoprofilaxis obstétrica y sus beneficios para la gestante

Obstetric psychoprofilaxis and its benefits for the pregnant

Revista Científica Mundo de la Investigación y el Conocimiento. Vol. 2 núm.3, julio, ISSN: 2588-073X, 2018, pp. 242-259

DOI: 10.26820/recimundo/2.(3).julio.2018.242-259

Editorial Saberes del Conocimiento

Recibido: 05/04/2018

Aceptado: 15/02/2018

Publicado: 30/07/2018

Correcnondencia: editnr(a) rerimunde rom
a. Universidad de Guayaquil; kerly.zambranom@ug.edu.ec
b. Universidad de Guayaquil; maria.torrezv@ug.edu.ec
c. Universidad de Guayaquil; anay.britom@ug.edu.ec
d. Universidad de Guayaquil; yesica.pazminom@ug.edu.ec 


\section{Psicoprofilaxis obstétrica y sus beneficios para la gestante}

Vol. 2, núm. 3., (2018)

Kerly Bachita Zambrano Macías; María Paulina Tórres Vasconez; Anay Teresita Brito Martínez; Yesica Yadira Pazmiño Mera

\section{RESUMEN}

Una de las principales causas de muerte a nivel mundial es la mortalidad materna, ante lo cual, la Organización Mundial de la Salud ha implementado una serie de planes con el objeto de trabajar mancomunadamente con sus países miembros. En este sentido, Ecuador a través de su Ministerio de Salud Pública a partir del 2014 crea la Norma Técnica para la Psicoprofilaxis Obstétrica y Estimulación Prenatal como herramienta fundamental en la lucha contra la reducción de Muertes Maternas. El presente trabajo se enfoca en los beneficios generales de la psicoprofilaxis obstétrica. La investigación se realizó mediante un estudio documental, donde se revisaron fuentes digitales veraces y actualizadas. Entre las principales bondades de la psicoprofilaxis obstétrica se encuentran la promoción del parto natural, la integración de la pareja y familia, el empoderamiento de la mujer y la reducción de la morbilidad y mortalidad materna y perinatal. El tema es de gran importancia para el fortalecimiento de los planes asociados ya que su conocimiento conducirá a las gestantes a una mayor participación, y por ende, tendrán menor probabilidad de una complicación durante su embarazo y parto y mayor probabilidad de un término feliz de su gestación y un recién nacido sano.

Palabras claves: Psicoprofilaxis, Obstétrica, Beneficios, Gestantes, Mortalidad. 


\title{
Psicoprofilaxis obstétrica y sus beneficios para la gestante
}

Vol. 2, núm. 3., (2018)

Kerly Bachita Zambrano Macías; María Paulina Tórres Vasconez; Anay Teresita Brito Martínez; Yesica Yadira Pazmiño Mera

\begin{abstract}
One of the main causes of death worldwide is maternal mortality, before which the World Health Organization has implemented a series of plans with the aim of working together with its member countries. In this sense, Ecuador through its Ministry of Public Health since 2014 creates the Technical Standard for Obstetric Prophylaxis and Prenatal Stimulation as a fundamental tool in the fight against the reduction of Maternal Deaths. The present work focuses on the general benefits of obstetric prophylaxis. The research was conducted through a documentary study, where true and updated digital sources were reviewed. Among the main benefits of obstetric psycho-prophylaxis are the promotion of natural birth, the integration of the couple and family, the empowerment of women and the reduction of maternal and perinatal morbidity and mortality. The issue is of great importance for the strengthening of the associated plans since their knowledge will lead the pregnant women to a greater participation, and therefore, they will be less likely of a complication during their pregnancy and childbirth and greater probability of a happy term of their pregnancy and a healthy newborn.
\end{abstract}

Keywords: Psychoprofilaxis, Obstetric, Benefits, Pregnant, Mortality. 


\section{Psicoprofilaxis obstétrica y sus beneficios para la gestante}

Vol. 2, núm. 3., (2018)

Kerly Bachita Zambrano Macías; María Paulina Tórres Vasconez; Anay Teresita Brito Martínez; Yesica Yadira Pazmiño Mera

\section{Introducción.}

El derecho de las mujeres a una vida sexual y reproductiva de calidad, a ejercer el sagrado derecho de dar vida sin presentar riesgos para su salud y la de su hijo, ha sido defendido y preservado por organismos como la Organización Mundial de la Salud (OMS) a nivel global. Los índices de mortalidad materna y perinatal a nivel mundial ha sido la lucha constante de la OMS para garantizar a las gestantes y sus hijos la calidad de vida que merecemos todos los seres humanos, más aún esta especial etapa de la reproducción humana. Dicho Organismo conjuntamente con los países miembros trabaja y vigilan esta causa de muerte alarmante en el mundo.

La (Organización Mundial de la Salud y Organización Panamericana de la Salud, 2011), establecen el Plan de Acción para Acelerar la Reducción de la Mortalidad Materna y la Morbilidad Materna Grave, cuyo objetivo principal es el siguiente:

Es un paso más para mejorar la salud de las mujeres, que contribuye indirectamente a los esfuerzos de los países para cumplir el Objetivo de Desarrollo del Milenio (OMD). Está relacionado con el Plan de acción regional para la reducción de la mortalidad materna en las Américas (1990), la resolución sobre "Población y Salud Reproductiva (1998), la Estrategia regional para la reducción de la mortalidad y la morbilidad maternas (2002), la Estrategia y plan de acción regionales sobre la salud del recién nacido en el contexto del proceso continuo de la atención de la madre, del recién nacido y del niño (2008), y el Plan de acción sobre la salud de los adolescentes y los jóvenes (2009). También aborda otros temas como hospitales seguros y redes integradas de servicios de salud (2009). (p. 5) 


\section{Psicoprofilaxis obstétrica y sus beneficios para la gestante}

Vol. 2, núm. 3., (2018)

Kerly Bachita Zambrano Macías; María Paulina Tórres Vasconez; Anay Teresita Brito Martínez; Yesica Yadira Pazmiño Mera

De lo anterior podemos destacar los diferentes planes de acción de la OMS ante la problemática de la mortalidad materna y la prestación de un servicio de salud digno tanto a las madres como a los recién nacidos, no sólo a través de planes individuales sino colectivos donde se integran a los diferentes países en una sola misión: reducir la morbilidad y mortalidad materna y perinatal. Es importante además, establecer que la meta a la que se refiere de los ODM 5 (Objetivos de Desarrollo del Milenium, de la Organización de Naciones Unidas, en su punto 5 relativo a la salud), se trata de la meta 5.A "Reducir en tres cuartas partes, entre 1990 y 2015, la mortalidad materna”. (Organización Mundial de la Salud, 2018)

El plan abarca un amplio espectro de puntos relacionados con la planta física de las instituciones que prestan servicios de salud, equipamiento e insumos, normas y procedimientos, dotación de talento humano, estadísticas vitales, instrumentos para registro, investigación y vigilancia de Muertes Maternas, análisis y uso de la información, sistema de notificación de Muertes Maternas, vigilancia de la morbilidad materna, atención humanizada y de calidad, participación comunitaria, barreras sociales y culturales de acceso a los servicios de salud, enfoque de derechos humanos, entre otros. (Organización Panamericana de la Salud, 2015)

El Plan Ecuador sin Muertes Maternas para su desarrollo establece cuatro ejes fundamentales: prevención y promoción; atención obstétrica; manejo estadístico, y auditorías de muerte materna. (Presidencia de la República del Ecuador, 2015). 


\section{Psicoprofilaxis obstétrica y sus beneficios para la gestante}

Vol. 2, núm. 3., (2018)

Kerly Bachita Zambrano Macías; María Paulina Tórres Vasconez; Anay Teresita Brito Martínez; Yesica Yadira Pazmiño Mera

En tal sentido, la psicoprofilaxis obstétrica es el proceso preventivo en esta materia que Ecuador ha decidido ejecutar.

Se entiende por psicoprofilaxis obstétrica, según (Ministerio de Salud del Perú, 2011):

Al proceso educativo para la preparación integral de la mujer gestante que le permita desarrollar hábitos y comportamientos saludables, así como una actitud positiva frente al embarazo, parto, puerperio, producto de la concepción y al recién nacido convirtiendo este proceso en una experiencia feliz y saludable, tanto para ella como para su bebe y su entorno familiar. Contribuye a la disminución de complicaciones obstétricas pre y pos natales al preparar física y psicológicamente a la gestante para un parto sin temor. Contribuye a la disminución de complicaciones perinatales al acortar el tiempo del proceso del parto y mejorar la oxigenación de la madre y el feto.

Para el (Ministerio de Salud Pública del Ecuador, 2014):

La Salud materna y perinatal constituye un importante indicador de las condiciones de desarrollo de un país, así como de la situación de su sector salud, siendo una prioridad, debido a que aun cuando la razón de mortalidad materna se ha reducido en los últimos años, existe la necesidad de contar con acciones que contribuyan con un mayor descenso de estas cifras, acorde con los Objetivos del Milenio. El Ministerio de Salud Pública, en el marco del cumplimiento de su rol de rectoría, regulación, planificación, coordinación, control y gestión de la Salud Pública 


\section{Psicoprofilaxis obstétrica y sus beneficios para la gestante}

Vol. 2, núm. 3., (2018)

Kerly Bachita Zambrano Macías; María Paulina Tórres Vasconez; Anay Teresita Brito Martínez; Yesica Yadira Pazmiño Mera

ecuatoriana, ha implementado diversas acciones encaminadas a la reducción de la morbilidad y mortalidad materna y perinatal, debido a sus altas tasas, por lo cual se inició el Plan para la Reducción de la Mortalidad Materna, que constituye un pilar fundamental dentro del Plan Nacional de la Salud y Derechos Sexuales y Reproductivos. (p. 7, 8)

En este sentido, nace el documento Norma Técnica de Psicoprofilaxis Obstétrica y Estimulación Prenatal, a través del cual el (Ministerio de Salud Pública del Ecuador, 2014) se plantea el siguiente objetivo:

Fortalecer el Sistema de Salud con la aplicación de esta normativa como un documento técnico metodológico que describa y guíe las acciones que deben cumplirse en las Unidades de Salud para brindar la Psicoprofilaxis Obstétrica y Estimulación Prenatal a las gestantes, como parte de su atención integral, con enfoque familiar, comunitario e intercultural, garantizando una atención de calidad y mejorando los indicadores de salud, contribuyendo con la reducción de la morbilidad y mortalidad materna y perinatal en el Ecuador. (p. 11)

El presente trabajo está orientado en plasmar los beneficios de la Psicoprofilaxis Obstétrica, según él (Ministerio de Salud Pública del Ecuador, 2014), establecidos en la Norma Técnica de Psicoprofilaxis Obstétrica y Estimulación Prenatal, en cuanto al "fortalecimiento de la atención del parto normal, la integración de la pareja y familia, el empoderamiento de la mujer y la reducción de la morbilidad y mortalidad materna y perinatal”. (p. 8) 


\section{Psicoprofilaxis obstétrica y sus beneficios para la gestante}

Vol. 2, núm. 3., (2018)

Kerly Bachita Zambrano Macías; María Paulina Tórres Vasconez; Anay Teresita Brito Martínez; Yesica Yadira Pazmiño Mera

Es importante el conocimiento de las bondades de la psicoprofilaxis, a objeto de fortalecer los planes de reducción de muerte materna, con énfasis en la educación y prevención y de esta forma garantizar tanto a las gestantes como a los recién nacidos, una mejor calidad de vida.

\section{Materiales y métodos.}

El presente estudio trata la Psicoprofilaxis Obstétrica de manera general, con un enfoque en los beneficios que su aplicación representa para las gestantes. La investigación va de lo general a lo específico, focalizándose en los principales planes de aplicación de la Psicoprofilaxis Obstétrica en Ecuador y sus beneficios generales, plasmando documentos importantes y actualizados con relación al estudio, así como cifras y datos de organismos públicos de dicha región como el Ministerio de Salud Pública, para finalizar con un análisis crítico de los beneficios de la aplicación de la Psicoprofilaxis Obstétrica en las gestantes, específicamente mediante los planes implementados por el sistema de salud del Ecuador.

El cuerpo de la investigación se desarrolló a partir de la recopilación y revisión de fuentes documentales digitales, considerando los principios de actualidad y veracidad, consultando páginas gubernamentales internacionales y nacionales oficiales, así como otros estudios académicos publicados en revistas especializadas y opiniones de expertos en la materia. 


\section{Psicoprofilaxis obstétrica y sus beneficios para la gestante}

Vol. 2, núm. 3., (2018)

Kerly Bachita Zambrano Macías; María Paulina Tórres Vasconez; Anay Teresita Brito Martínez; Yesica Yadira Pazmiño Mera

\section{Resultados.}

Beneficios de la Psicoprofilaxis Obstétrica para la Gestante

Son múltiples los beneficios de la psicoprofilaxis obstétrica, el Centro de Psicoprofilaxis, Estimulación Prenatal y Temprana (Wawakumara, 2013), cita a la Organización Mundial de la Salud (OMS) y otras importantes instituciones internacionales, recomiendan la Psicoprofilaxis Obstétrica, por sus efectos positivos y beneficiosos. Asimismo, acerca de estos beneficios para la gestante menciona los siguientes:

Menor grado de ansiedad, permitiéndole adquirir y manifestar una actitud positiva. Adecuada respuesta durante las contracciones uterinas, utilizando adecuadamente las técnicas de relajación, respiración, masajes y demás. Mayor posibilidad de ingresar al centro de atención, en trabajo de parto, con una dilatación más avanzada. Evitar innecesarias y repetidas evaluaciones previas al ingreso para la atención de su parto. Menor duración de la fase de dilatación en relación al promedio normal. Menor duración de la fase expulsiva. Menor duración total del trabajo de parto. Esfuerzo de la madre más eficaz, sin perder atención al objetivo y significado de su labor. Menor uso de fármacos en general. Menor uso de analgésicos. Menor riesgo a presentar complicaciones obstétricas. Acusará menor malestar y / o dolor durante el trabajo de parto, bajo el fundamento de saber aplicar toda su fuerza física, equilibrio mental y capacidad de concentración en miras de controlar la situación, entre otras. 


\section{Psicoprofilaxis obstétrica y sus beneficios para la gestante}

Vol. 2, núm. 3., (2018)

Kerly Bachita Zambrano Macías; María Paulina Tórres Vasconez; Anay Teresita Brito Martínez; Yesica Yadira Pazmiño Mera

El (Ministerio de Salud Pública del Ecuador, 2014) a través de su Norma Técnica de Psicoprofilaxis y Estimulación Prenatal, engloba los beneficios de la Psicoprofilaxis en cuatro grupos: la promoción del parto natural, integración de la pareja y la familia al proceso, empoderamiento de la mujer y la reducción de la morbilidad y la mortalidad materna y perinatal.

\section{Promueve el parto natural}

Katiuska Hernández, gerente del Hospital General Guasmo Sur (HGGS), en una reseña de (El Telégrafo, 2017), acerca de las cesáreas recordó:

Existe un acuerdo ministerial en el sector de la salud, público y privado, en el que se dispone que el médico especialista justifique el motivo por el cual practicará una cesárea a una paciente. "No se trata de que la madre escoja dar a luz mediante cesárea. Es el profesional quien debe decidir y sustentar las razones médicas". Agregó, que las madres optan por la cesárea "porque creen en muchos mitos sobre el parto natural, cuando este es más beneficioso para la madre".

En tal sentido, por medio de la psicoprofilaxis obstétrica se promueve el parto natural, es importante que las gestantes sean conscientes de los beneficios del mismo, y esto es lo que se desea en Ecuador, es el logro que persiguen los planes de acción donde se aplican estas normas técnicas, ya sea a través de clubes, talleres individuales o grupales, se debe informar a la gestante acerca de todo lo relacionado con su gestación y parto, incluso acerca de los cuidados del recién 


\section{Psicoprofilaxis obstétrica y sus beneficios para la gestante}

Vol. 2, núm. 3., (2018)

Kerly Bachita Zambrano Macías; María Paulina Tórres Vasconez; Anay Teresita Brito Martínez; Yesica Yadira Pazmiño Mera

nacido, de esta forma será consciente de tomar las mejores decisiones para su salud y la de su hijo, y ejercerá su empoderamiento al dialogar con los profesionales de la salud y elegir el parto natural, si no hay ningún impedimento para el mismo.

Es importante resaltar los beneficios que brinda un parto natural para la madre, el recién nacido, incluso para la pareja, (Zabala, 2014) menciona entre las principales:

Al nacer en un parto natural o psicoprofiláctico, el bebé se beneficia principalmente en la oxigenación que recibe en la primera respiración, ya que las contracciones del trabajo de parto lo estimulan de tal forma que puede respirar mejor en el momento del nacimiento y estar más activo los primeros días de vida. La mujer que ha tenido un parto natural garantiza un vínculo estrecho con su bebé y facilita el proceso de la lactancia, ya que la experiencia de su participación activa la dispone a un estado emocional óptimo, lo que favorece positivamente la nueva relación, provocando que la madre no quiera separarse de su bebé. Este tipo de partos es un proceso que vive la mujer con su pareja desde el embarazo, y el apoyo que éste les brinda, les permite crecer de pareja conyugal a pareja parental. La presencia de la pareja en el nacimiento favorece el apego y la formación del vínculo padre-madre-hijo.

\section{Integración Pareja/Familia}

Por otra parte, la Norma Técnica de Psicoprofilaxis del (Ministerio de Salud Pública del Ecuador, 2014), pretende con respecto a la pareja y la familia de la gestante: 


\section{Psicoprofilaxis obstétrica y sus beneficios para la gestante}

Vol. 2, núm. 3., (2018)

Kerly Bachita Zambrano Macías; María Paulina Tórres Vasconez; Anay Teresita Brito Martínez; Yesica Yadira Pazmiño Mera

Se fomentará la participación activa de la pareja durante toda la jornada obstétrica (gestación, parto y posparto), contribuyendo al manejo adecuado de los temores o rechazo a las molestias y/o dolores que se presentan durante la gestación y el trabajo de parto, cuyo conjunto de reacciones negativas podrían dificultar su normal proceso. Por ello existen un conjunto de objetivos, criterios y procesos que conforman las sesiones de Psicoprofilaxis Obstétrica y Estimulación Prenatal, para ser brindadas a la gestante y su pareja, lo cual se proyecta en un buen eje de prevención y promoción de estilos de vida saludable para la familia y comunidad. (p. 7)

Asimismo, con respecto a dicha norma, entre sus objetivos establece:

Brindar a la gestante y su pareja una preparación integral, teórica, física y psico-afectiva con enfoque familiar, comunitario e intercultural. Describir los contenidos de las sesiones de Psicoprofilaxis Obstétrica y Estimulación Prenatal que preparen integralmente a la gestante y su pareja, además de fortalecer el vínculo afectivo prenatal entre los padres y respecto de la llegada de su hijo. Identificar las acciones más costo-efectivas para mejorar la calidad de atención a gestantes y la participación activa de la pareja, la familia y la comunidad. (Ministerio de Salud Pública del Ecuador, 2014)

Es importante en el proceso de Psicoprofilaxis Obstétrica integrar a la pareja de la gestante, principalmente, también puede integrarse cualquier otro familiar cercano, que brinde la confianza y el apoyo suficiente a la madre durante el embarazo, parto y cuidados del recién nacido, de esta forma se garantiza un mejor desarrollo de la gestación, mejores resultados en los 


\section{Psicoprofilaxis obstétrica y sus beneficios para la gestante}

Vol. 2, núm. 3., (2018)

Kerly Bachita Zambrano Macías; María Paulina Tórres Vasconez; Anay Teresita Brito Martínez; Yesica Yadira Pazmiño Mera

trabajos de parto y mayores probabilidades de una buena salud para la madre y el recién nacido, fomenta los vínculos familiares y todo ello representa mejoras al momento de la atención para el personal de salud, ya que disminuye el número de complicaciones.

\section{Empoderamiento de la Mujer}

El empoderamiento supone una base sólida para una maternidad feliz, según (Carabantes, 2014), las gestantes deben decir acerca del empoderamiento que:

Es hacerse cargo de su salud y buscar su bienestar. Tomar decisiones al respecto, utilizar la capacidad de razonamiento y de libertad. Es preguntarse qué se desea. Para ello, deben recoger información, observar las diferentes posibilidades, compartir sus decisiones, elaborar el plan de acción y ponerlos en marcha. Los resultados deben ser evaluados para valorar si se repiten en el camino elegido o si deben ser cambiados.

En este orden de ideas, la aplicación de la psicoprofilaxis obstétrica, según el (Ministerio de Salud Pública del Ecuador, 2014), por medio de su norma técnica tiene como propósito:

Devolver el rol protagónico a la madre, con el acompañamiento de su pareja y familia, en un contexto de estilos de vida saludables y con un enfoque de respeto a sus derechos y patrones culturales, propiciando y consolidando un estrecho vínculo afectivo prenatal, como cimiento para el "nido" familiar, es decir, el ambiente propicio para el desarrollo físico, afectivo, intelectual y 


\section{Psicoprofilaxis obstétrica y sus beneficios para la gestante}

Vol. 2, núm. 3., (2018)

Kerly Bachita Zambrano Macías; María Paulina Tórres Vasconez; Anay Teresita Brito Martínez; Yesica Yadira Pazmiño Mera

social del hijo, fortalecido por la acogida y afecto de los padres, lo cual se traducirá en su bienestar general, favorable a su crecimiento y desarrollo desde su gestación. (p. 6)

\section{Reducción de la Morbilidad y la Mortalidad Materna y Perinatal.}

La iniciativa de la creación de la Norma Técnica de Psicoprofilaxis Obstétrica y Estimulación Prenatal, nace a partir de resultados en algunos centros de salud de Ecuador, donde ya se habían aplicado cursos de psicoprofilaxis a las gestantes y sus parejas o familiares, con excelentes resultados para la disminución de las muertes maternas y perinatales, estas prácticas previas, según (Ministerio de Salud Pública del Ecuador, 2014) consiguieron:

Potenciar la atención integral de la mujer desde la concepción hasta la etapa postnatal, incrementándose la cobertura de atención a las gestantes adolescentes, como parte de los objetivos, a partir del equipo de Obstetrices como el recurso humano profesional idóneo para ser un eslabón significativo entre la población y la atención primaria, traduciéndose en una estrategia que contribuye con la reducción de las cesáreas y de la morbilidad y mortalidad materna y perinatal, constituyéndose así en un Eje de la Prevención Prenatal. (p. 8)

Estos resultados impulsaron los planes de acción que Ecuador tiene en la actualidad, en donde a través de la psicoprofilaxis como medio de educación de la gestante y sus familiares, como medio preventivo, se mantiene la lucha contra la morbilidad y mortalidad materna y perinatal. 


\section{Psicoprofilaxis obstétrica y sus beneficios para la gestante}

Vol. 2, núm. 3., (2018)

Kerly Bachita Zambrano Macías; María Paulina Tórres Vasconez; Anay Teresita Brito Martínez; Yesica Yadira Pazmiño Mera

Según datos registrados por él (Ministerio de Salud Pública del Ecuador, 2017), tomando el periodo 2017 - 2014 (considerando que este último fue el año de puesta en marcha de la norma técnica de profilaxis), para el 2017 se registraron hasta la semana epidemiológica (SE) 50 un total de 127 muertes maternas (MM), en el 2016 se registraron 124 MM, para el 2015 fueron $148 \mathrm{MM}$ y en el 2014 un total de $156 \mathrm{MM}$, de lo que se desprende lo siguiente:

Comparando el año 2014 con el 2017 hasta la SE 50, en el 2017 hay una disminución del 18,59\% (29 casos). Comparando el año 2015 con el 2017 hasta la SE 50, en el 2017 hay una disminución del 14,19\% (21 casos). Comparando el año 2016 con el 2017 hasta la SE 50, en el 2017 hay un incremento del 2,36\% (3 casos).

Tomando en consideración las estadísticas más recientes del (Ministerio de Salud Pública del Ecuador, 2018), hasta la semana epidemiológica (SE) 23 de lo que va de año, se han registrado (hasta los 42 días de puerperio) 59 muertes maternas (MM), para el 2017 fueron 58 MM y 65 MM para el 2016, igualmente hasta la SE 23, de lo que se deriva:

Comparando el año 2016 con el 2018 hasta la SE 23, en el 2018 hay una disminución del 9,68\% (6MM). Comparando el año 2017 con el 2018 hasta la SE 23, en el 2018 hay un aumento $\operatorname{del} 5,36 \%(1 \mathrm{MM})$.

En cuanto al estado del producto al nacimiento, según estadísticas del (Ministerio de Salud Pública del Ecuador, 2018), hasta la semana epidemiológica (SE) 23: 


\section{Psicoprofilaxis obstétrica y sus beneficios para la gestante}

Vol. 2, núm. 3., (2018)

Kerly Bachita Zambrano Macías; María Paulina Tórres Vasconez; Anay Teresita Brito Martínez; Yesica Yadira Pazmiño Mera

De las 23 muertes maternas (MM) que su parto fue por Cesárea, el 78,26\% de los niños vivieron y el 21,74\% de los niños fallecieron. De las $18 \mathrm{MM}$ que su parto fue vaginal, el $83,33 \%$ de los niños vivieron y el $16,64 \%$ de los niños fallecieron. Se concluye que de las 41MM que su parto fue por cesárea o parto vaginal el $80,49 \%$ (33RN) vivieron y se encuentran en custodia del padre o de familiares y el 19,51\% (8RN) fallecieron.

\section{Conclusiones.}

Son múltiples los beneficios de la aplicación de la Psicoprofilaxis Obstétrica para las gestantes, por cuanto representa una herramienta de educación no sólo para la madre sino para su pareja y familiares cercanos. Abarca desde el momento de la concepción, pasando por el parto, hasta los cuidados del recién nacido y se constituye como uno de los principales medios de prevención de morbilidad y muertes maternas y perinatales.

En Ecuador, estas técnicas psicoprofilácticas han sido mejoradas a través del tiempo, a partir de 2014 se crea la Norma Técnica para la Psicoprofilaxis Obstétrica y la Estimulación Prenatal, con el propósito fundamental de brindar a la gestante las herramientas para un embarazo, parto y cuidados del recién nacido, de manera feliz y garantizando las menores probabilidades de riesgos tanto para ella como para el producto durante todas las etapas del proceso. 


\section{Psicoprofilaxis obstétrica y sus beneficios para la gestante}

Vol. 2, núm. 3., (2018)

Kerly Bachita Zambrano Macías; María Paulina Tórres Vasconez; Anay Teresita Brito Martínez; Yesica Yadira Pazmiño Mera

El parto natural es uno de los principales beneficios que puede obtener la gestante de la psicoprofilaxis, manejar la información que le permita participar en las mejores decisiones para ella y el recién nacido, durante este proceso, es vital para la obtención de los mejores resultados.

La gestante es la protagonista y como tal debe estar en pleno conocimiento y conciencia de lo que le sucede y lo que es mejor para su salud y la de su hijo. La integración de la pareja y la familia a este proceso es fundamental, ya que coadyuvan con la gestante y el equipo de salud en cada una de las etapas. El fin último de los planes de psicoprofilaxis es la disminución de las complicaciones y de muertes maternas y de recién nacidos, meta con la cual ha contribuido eficientemente el Ministerio de Salud del Ecuador según lo interpretado en las estadísticas de muertes maternas desde el 2014 (año de la puesta en marcha de la referida norma técnica y sus planes de acción), los cuales han reflejado un descenso de aproximadamente un $18 \%$ de muertes maternas, que si bien es cierto que no alcanza las metas propuestas por los organismos internacionales y nacionales en materia de salud, no es menos cierto que representa un meritorio avance, que aumenta la garantía para la gestante de un proceso feliz y una mejor calidad de vida para ella y el recién nacido.

\section{Bibliografía.}

Carabantes, A. (2014). Todo Maternidad. Recuperado el 16 de Julio de 2018, de http://www.todomaternidad.com/informacion/nuestros-profesionales/la-comadrona/354empoderamiento-de-la-mujer-en-el-embarazo

El Telégrafo. (05 de Septiembre de 2017). El Telégrafo. Recuperado el 15 de Julio de 2018, de https://www.eltelegrafo.com.ec/noticias/sociedad/6/las-gestantes-aprenden-a-cuidarse-enun-club 


\section{Psicoprofilaxis obstétrica y sus beneficios para la gestante}

Vol. 2, núm. 3., (2018)

Kerly Bachita Zambrano Macías; María Paulina Tórres Vasconez; Anay Teresita Brito Martínez; Yesica Yadira Pazmiño Mera

Ministerio de Salud del Perú. (06 de Mayo de 2011). minsa.gob.pe. Recuperado el 14 de Julio de 2018, de ftp://ftp2.minsa.gob.pe/normaslegales/2011/RM361-2011-MINSA.pdf

Ministerio de Salud Pública del Ecuador. (Septiembre de 2014). aplicaciones.msp.gob.ec. Recuperado el 15 de Julio de 2018, de https://aplicaciones.msp.gob.ec/salud/archivosdigitales/sigobito/tareas_seguimiento/722/ PROPUESTA\%20NORMA\%20PSICOPROFILAXIS\%20OBSTETRICA\%20Y\%20EST IMULACION\%20PRENATAL\%20-\%204.pdf

Ministerio de Salud Pública del Ecuador. (2017). salud.gob.ec. Recuperado el 15 de Julio de 2018, de https://www.salud.gob.ec/wp-content/uploads/2017/07/Gaceta-MM-SE-50.pdf

Ministerio de Salud Pública del Ecuador. (2018). salud.gob.ec. Recuperado el 15 de Julio de 2018, de https://www.salud.gob.ec/wp-content/uploads/2017/07/Gaceta-de-MM-SE23.pdf

Organización Mundial de la Salud. (19 de febrero de 2018). Organización Mundial de la Salud. Recuperado el 02 de junio de 2018, de http://www.who.int/es/news-room/factsheets/detail/millennium-development-goals-(mdgs)

Organización Mundial de la Salud y Organización Panamericana de la Salud. (2011). Plan de acción para acelerar la reducción de la mortalidad materna y la morbilidad materna grave. Washington. Recuperado el 02 de junio de 2018, de https://www.paho.org/clap/index.php?option=com_docman\&view=download\&category_ slug=publicaciones\&alias=267-plan-de-accion-para-acelerar-la-reduccion-de-lamortalidad-materna-y-la-morbilidad-materna-grave-cd51-12\&Itemid=219\&lang=en

Organización Panamericana de la Salud. (2015). paho.org. (O. P. Salud, Ed.) Recuperado el 02 de junio de 2018, de https://www.paho.org/clap/index.php?option=com_docman\&view=download\&category _ slug=documentos-de-noticias \&alias $=473$-implementacion-planes-de-reduccion-de-lamorbi-mortalidad-materna-ecuador\&Itemid=219\&lang=es

Presidencia de la República del Ecuador. (2015). presidencia.gob.velecuador. Recuperado el 02 de junio de 2018, de https://www.presidencia.gob.ec/ecuador-radicalizara-la-luchacontra-la-mortalidad-materna/

Wawakumara. (13 de Julio de 2013). Wawakumar. Recuperado el 14 de Julio de 2018, de http://www.wawakumara.com.pe/ventajas-de-la-psicoprofilaxis-obstetrica-oms/

Zabala, J. O. (2014). Ginecología militar. Recuperado el 16 de Julio de 2018, de http://ginecomilitar.com/beneficios-del-parto-psicoprofilactico 